\title{
A STUDY ON ELECTRICAL PERFORMANCES AND LIFETIME OF A FLEXIBLE ELECTROCHROMIC TEXTILE DEVICE
}

\author{
Constance Moretti ${ }^{1,2 *}$, Xuyuan Tao ${ }^{1,2}$, Vladan Koncar ${ }^{1,2}$, Ludovic Koehl ${ }^{1,2}$ \\ ${ }^{1}$ Gemtex, Ensait, 2 allée Louise et Victoire Champier, Roubaix 59100, France \\ ${ }^{2}$ Univ. Lille Nord de France, Lille 59000, France \\ e-mail: constance.moretti@ensait.fr
}

\begin{abstract}
:
Using their ability to change their color according to an external stimulation, chromic materials can be used to form a color-changing textile. Electrochromism, more particularly, is a colour change phenomenon caused by the application of an electrical potential. A flexible textile electrochromic device composed of four layers is presented. In order to improve the lifetime of this structure, the electrical performances of the electrolyte layer are studied. $A$ method to measure and calculate the resistance variations of the electrolyte applied on a textile cotton substrate is given. Relations between the electrical performances of the electrolyte and the electrochromic effect of the device are also highlighted.
\end{abstract}

\section{Keywords:}

Smart textile, electrochromism, flexible display, electrolyte, sol-gel, conductivity

\section{Introduction}

Intelligent textiles can be defined as textiles that are able to sense and analyse a signal and respond and adapt to it in an intelligent way [1]. Our clothing may be considered as a medium and an interface between us and the environment. Considering the large possibilities offered by these new materials, extraordinary applications can be imagined. One of them is the idea of a colour-changing textile. To this end, chromic materials can be very useful. Chromism is the ability of a material to change its colour according to a stimulus [2]. Colour-changing textiles have various and high value potential applications. Nevertheless, their lifetime is a key issue that may limit their potential. The main objective of this article is to study this lifetime in order to get a better understanding of phenomena related to it and possibly to improve this performance in the future. This colour change occurs either from one colour to another or from a colourless state to a coloured state. Moreover, chromic phenomena are reversible. Different chromisms are defined after the different stimuli that cause the change, such as thermochromism for change caused by temperature, photochromism for a change caused by light or electrochromism for the ability of a material to change its optical properties when an appropriate electrical potential is applied across it [3].

The optical properties of the electrochromic material are linked to its oxidation state and hence can be manipulated by the oxidation-reduction process through the loss or gain of electrons number. The required voltage for an electrochromic colour change is very low. The colour remains even when the current has ceased to flow (the so-called «memory effect» [4]) and the colour change is reversible when the inverted potential is applied. Existing applications of the electrochromic materials are mostly in a range of automotive and architectural industries, e.g. rear-view mirrors for cars and smart windows. Studies [5] show that the use of an electrochromic smart window can save up to $50 \%$ of the energy used for air conditioning per year. The advantages of electrochromism are its large colour palette [6], very low amount of energy required and visible colour change in every position.

Generally, an electrochromic device is composed of seven layers, as seen in figure 1a [7]. Glass is used as a mechanical support. Two electrodes provide electricity to the structure; at least one of them has to be transparent in order to allow the colour change to be visible. The storage film contains ions, which then are labile in the electrolyte. The electrochromic layer is the colour-changing component. Recent projects undertaken by the GEMTEX laboratory [8-10] have led to the development of a new electrochromic structure, whose number of layers has been reduced to four, as seen in figure $1 \mathrm{~b}$. In order to get a flexible structure, a flexible plastic layer coated with a conductive polymer can be used. Because of its electrically conductive properties, in addition to good transparency, polyester thin film coated with indium tin oxide (PET-ITO) has been chosen for our structure. The electrochromic material is mixed with the electrolyte, and the solution is stored in a textile substrate.

The lifetime of electrochromism is one of the most important factors that need to be improved, and therefore understood, in order to allow its larger diffusion and use. A fast degradation of the electrochromic effect of our textile device has already been observed using colorimetric measurements in the CIE $L^{*}, a^{*}$ and $b^{*}$ space. Hence, we voice the hypothesis that $a$ degradation of the electrolyte properties may participate in the degradation of the colour change, through a loss of conductivity. 


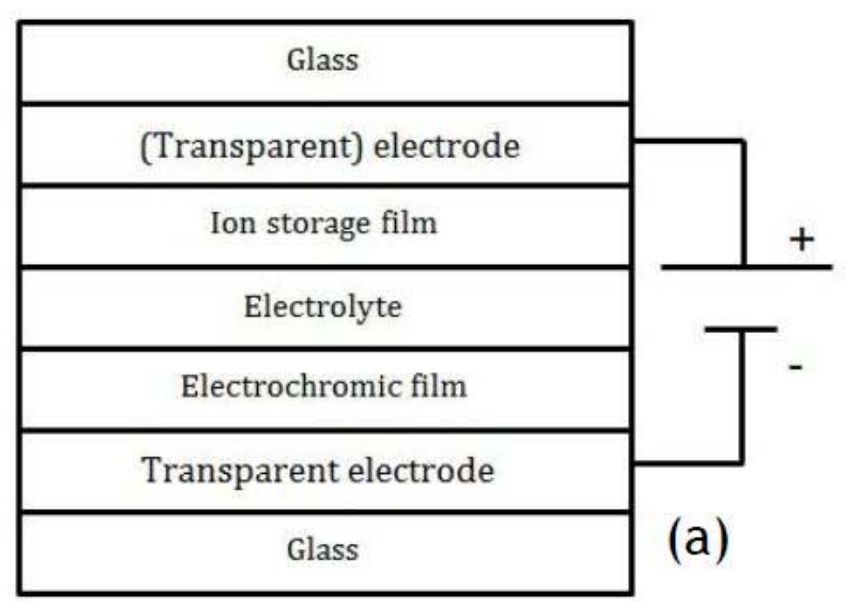

Figure 1. Rigid (a) and flexible (b) electrochromic devices

To this purpose, the present article explicates the study made on the electrical performances of the electrolyte used in a fourlayer electrochromic textile device.

\section{Experimental}

\section{Materials and methods}

For every structure, a $100 \%$ cotton white fabric $\left(112 \mathrm{~g} / \mathrm{m}^{2}\right)$ provided by Tissus Hallynck in Roubaix (FR) was used as the textile substrate. The method proposed by Ding et al. [11] was used to prepare an electrolyte that can be photo-polymerised to form a sol-gel. The electrolyte solution is composed of $10 \mathrm{~g}$ of propylene carbonate, $10 \mathrm{~g}$ of poly(ethyleneglycol) diacrylate $\left(\mathrm{M}_{\mathrm{n}}=700\right), 2 \mathrm{~g}$ of lithium trifluoromethanesulphonate and $35 \mathrm{mg}$ of 2,2-dimethoxy-2-phenyl-acetophenone. All chemicals were purchased from Sigma-Aldrich. The electrochromic monomer dithieno(3,2-b : 2',3'-d)thiophene (DTn(3,2-b:2',3'-d)Th) $\left(\mathrm{C}_{8} \mathrm{H}_{4} \mathrm{~S}_{3}, 97 \%\right)$ and PET-ITO films, with a surface resistivity of $60 \Omega / s q$, were purchased from Sigma-Aldrich. In order to eliminate the influence of the electrochromic material, a structure using the pure electrolyte, without electrochromic material, was employed. A homogeneous surface contact between the electrolytic solution applied into the textile substrate and the electrode is obtained when the textile is saturated with the solution: around $400 \mathrm{~g} / \mathrm{m}^{2}$ was applied. The photo-polymerisation of the electrolyte was carried out in a light box (VeriVide CAC 60, UV light $18 \mathrm{~W}$ ) for 15 minutes, $6 \mathrm{~cm}$ away from the lamp, at room temperature. To form an electrochromic device, $2.5 \mathrm{wt} \%$ of the electrochromic monomer powder is mixed with the electrolyte and applied by pipette in the textile cotton substrate. The mixture diffuses in the textile substrate. The electrochromic monomer is then electrically polymerised by applying $+3 \mathrm{~V}$ for 30 seconds across it.

We voice the hypothesis that the electrolyte has a variable resistance, dependant of the electrical potential applied across it and the length of this application. In order to get perfectly reliable data, the electrical resistance of the electrolyte is not directly measured. A constant resistance is inserted in the circuit. The emitted potential and the potential across this constant resistance are measured, as seen in figure 2. From $V_{E L}$

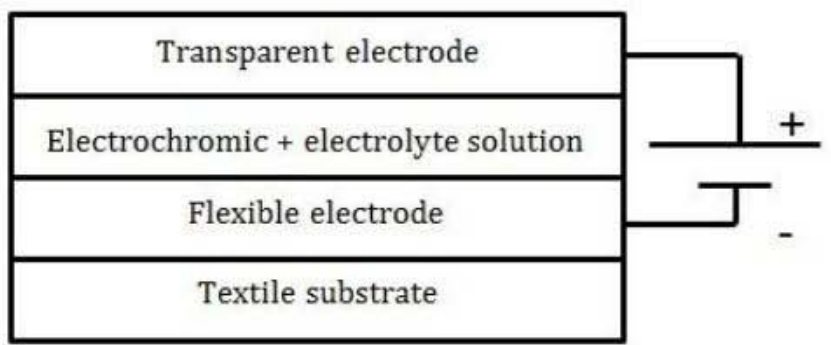

(b)

the potential across the electrolyte, $V_{G}$ the potential applied by the generator, $V_{R}$ the potential across the constant resistance and $i$ the current, we have:

$$
V_{E L}=V_{G}-V_{R}(1)
$$

So, according to Ohm's law:

$$
\frac{V_{E L}}{V_{R}}=\frac{R_{E L} \times i}{R_{R} \times i} \quad \Rightarrow \quad R_{E L}=R_{R} \times \frac{V_{E L}}{V_{R}}(2)
$$

A signal alternatively switching from a positive $(+5 \mathrm{~V})$ to negative $(-5 \mathrm{~V})$ potential values every 30 seconds is sent by the generator. The variation of the generator and constant resistance potentials are measured using a Keithley KUSB 3100 data acquisition module by Keithley Instrument.

To measure the influence of photo-polymerisation on colour, colorimetry measurements have been carried out with a spectrophotometer (Spectraflash SF600 Plus by Data Color International). A signal alternatively switching from a positive $(+5 \mathrm{~V})$ to negative $(-5 \mathrm{~V})$ potential value every 30 seconds is sent to the measured electrochromic device. Automatic colour

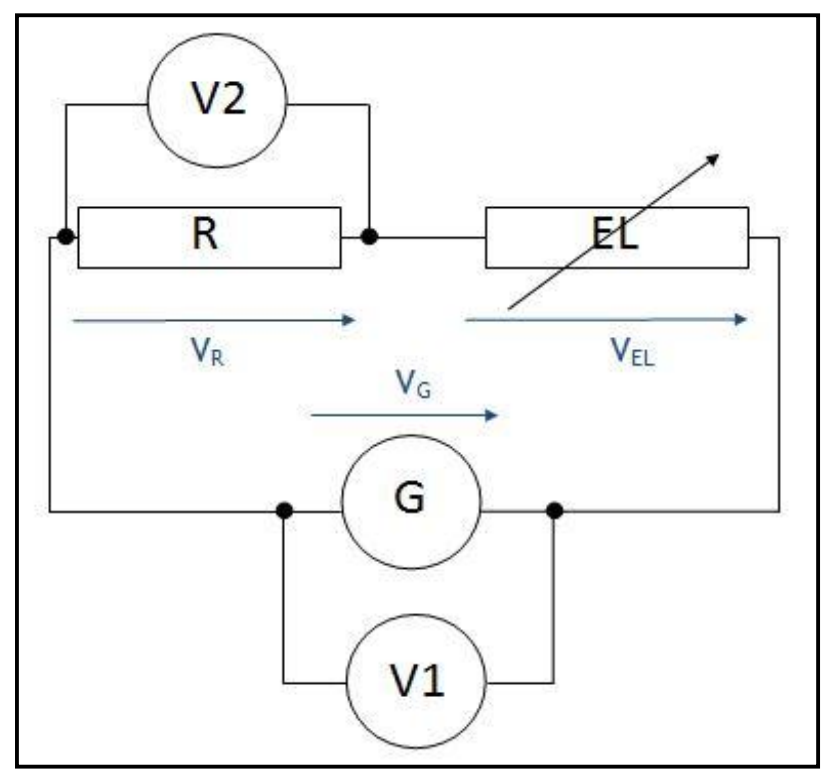

Figure 2. Electrical circuit 
measurements are carried out with a measurement frequency of $0.2 \mathrm{~Hz}$. The $L^{*}, a^{*}$ and $b^{*}$ coordinates have been analysed in the CIE $L^{*}, a^{*}$ and $b^{*}$ space.

In the CIE $L^{*}, a^{*}$ and $b^{*}$ space, Cartesian coordinates $L^{*}, a^{*}$ and $b^{*}$ represent three components of the colour. $L^{*}$ is the lightness coordinate from $0 \%$ (black) to $100 \%$ (white); $a^{*}$ and $b^{*}$ coordinates are colour-opponent dimensions: from green $\left(-a^{*}\right)$ to red $\left(+a^{*}\right)$ for the $a^{*}$ axis and from blue $\left(-b^{*}\right)$ to yellow $\left(+b^{*}\right)$ for the $b^{*}$ axis. Every colour can be represented by one point. A colour change between two points is then logically a segment and can by calculated by:

$\operatorname{DeltaE} C I E(L * a * b *)_{A-B}=\sqrt{\left(L *_{A}-L *_{B}\right)^{2}+\left(a *_{A}-a *_{B}\right)^{2}+\left(b *_{A}-b *_{B}\right)^{2}}(3)$

\section{Results and discussion}

\section{$\underline{\text { Colorimetric measurements }}$}

\section{Ageing of the electrochromic effect}

Figure $3 a$ and $3 b$ represent the CIE $L^{*}, a^{*}$ and $b^{*}$ colour variations of the electrochromic textile device when $\pm 5 \mathrm{~V}$ are applied successively every 30 seconds. The oxidised state of the textile electrochromic device is initially green, and the reduced state is initially red. However, as observed in figure $3 \mathrm{~b}$, after 50 minutes of test, both states are darker and browner. The obtained colours are darker ( $L^{*}$ decreasing) and less intense ( $\left|a^{*}\right|$ and $\left|b^{*}\right|$ decreasing). The $a^{*}$ coordinate, representing the green/red variations, remains positive after the first cycles, which means that the colour turns more red than green. Finally, the difference in colour between the oxidised and reduced states is, importantly, decreasing. Multiple parameters may influence this colour degradation. Here, the influence of the electrolyte electric properties will be observed.

\section{Electrical measurements}

\section{Capacitor behaviour of the electrolyte}

Figure 4 presents the evolution of the electric potential for the generator, constant resistance $(R)$ and electrolyte $(E L)$. The constant resistance of the circuit is $R=1.435 \mathrm{M} \Omega$. It has been chosen after preliminary experiments to be in the same order of resistance value of the electrolyte. The observed potential across the electrolyte does not instantly follow the generator potential. This phenomenon can be explained by the formation of electric double layers on the both faces of the electrolyte [12].

An interesting point that needs to be explained is the fact that at every potential switch (from $+5 \mathrm{~V}$ to $-5 \mathrm{~V}$ or from $-5 \mathrm{~V}$ to $+5 \mathrm{~V})$, the constant resistance potential is superior to the emitted potential for approximately 5 seconds (see point $A$ in figure 4). This is due to the potential difference between the
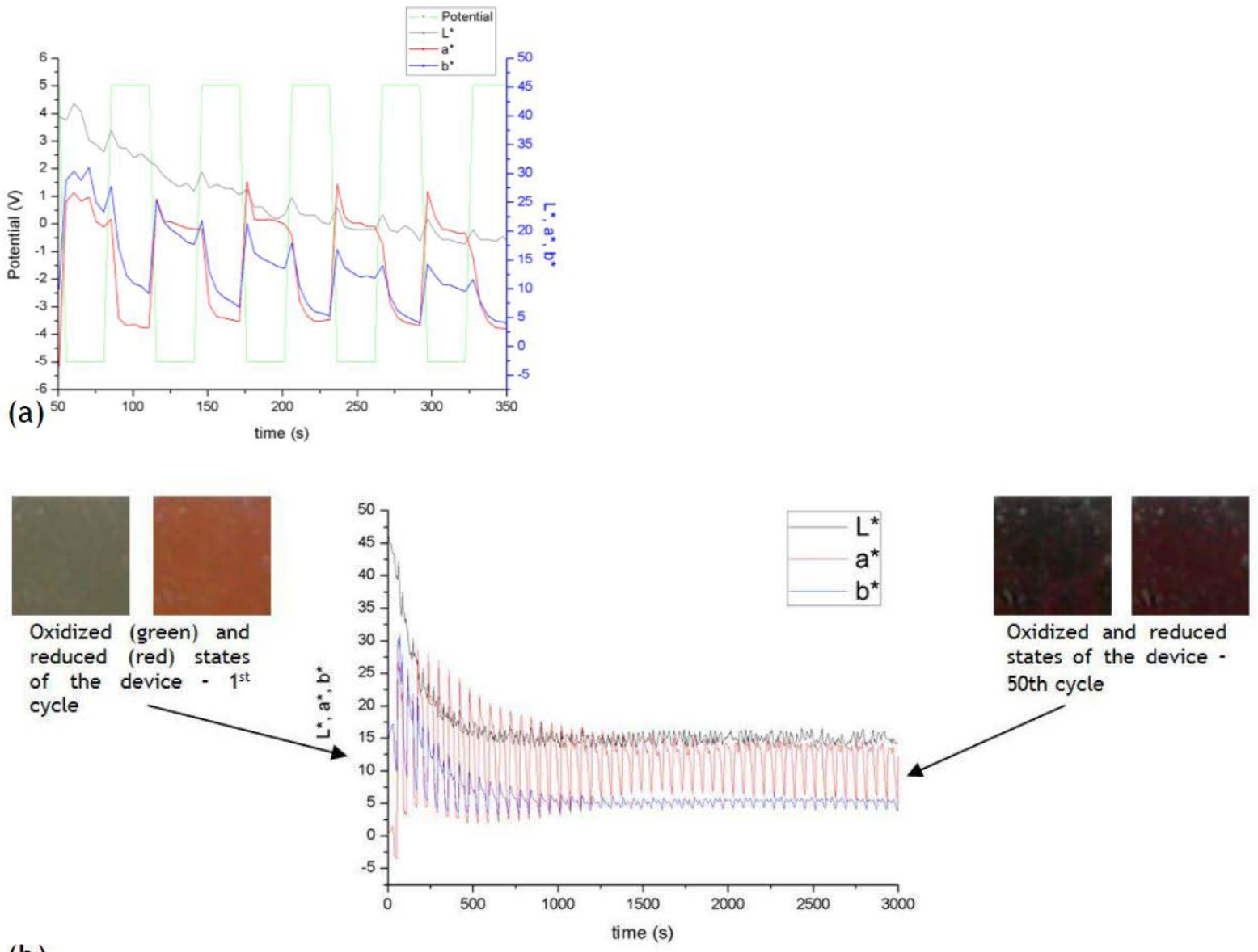

(b)

Figure 3. $L^{*}, a^{*}$ and $b^{*}$ coordinates of the colour of the EC device using DTn(3,2-b:2', $\left.3^{\prime}-d\right)$ Th (a) for 5 cycles and (b) for 50 cycles 


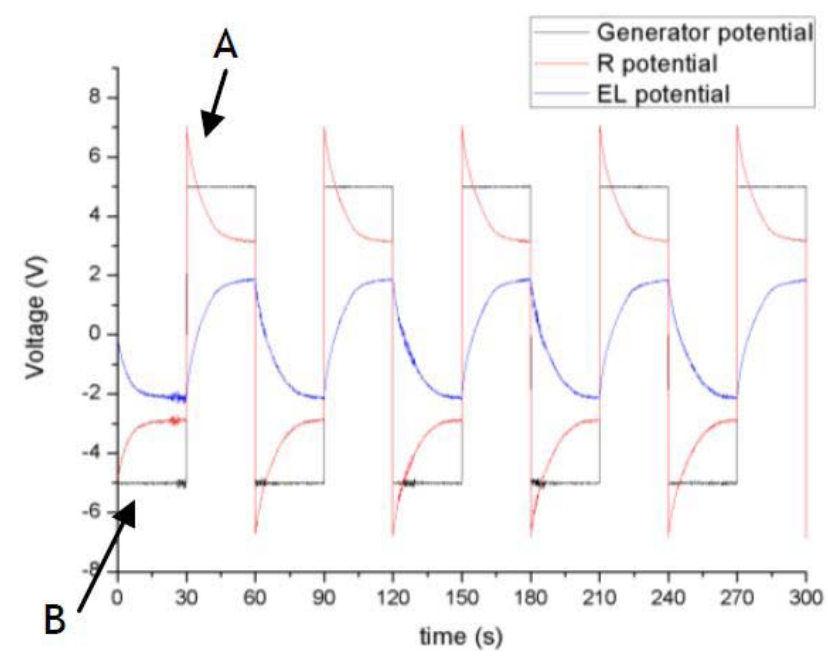

Figure 4. Potentials with the electrolyte

electrode and the electrolyte. The potential difference between the negatively charged electrode (excess of electrons) and the cations at one electrode, and the positively charged electrode (lack of electrons) and the anions at the other electrode is added to the potential emitted by the generator. For the first half-cycle, when the electrolyte-capacitor is not charged yet, the constant resistance potential is indeed below $-5 \mathrm{~V}$ (see point $B$ in figure 4).

To ascertain that the capacitor effect effectively comes from the electrolyte, the same measurement was carried out, replacing the electrolyte by a constant resistance $\mathrm{R} 2=200 \mathrm{k} \Omega$ and using a constant resistance in the circuit $R 1=400 \mathrm{k} \Omega$ instead of $R$. Results indicated in figure 5 confirm that the capacitor effect is from the electrolyte.

The time for total charge and discharge of the electrolytecapacitor is measured by applying a constant positive (for charge) or negative (for discharge) voltage across it and is approximately 3 minutes.

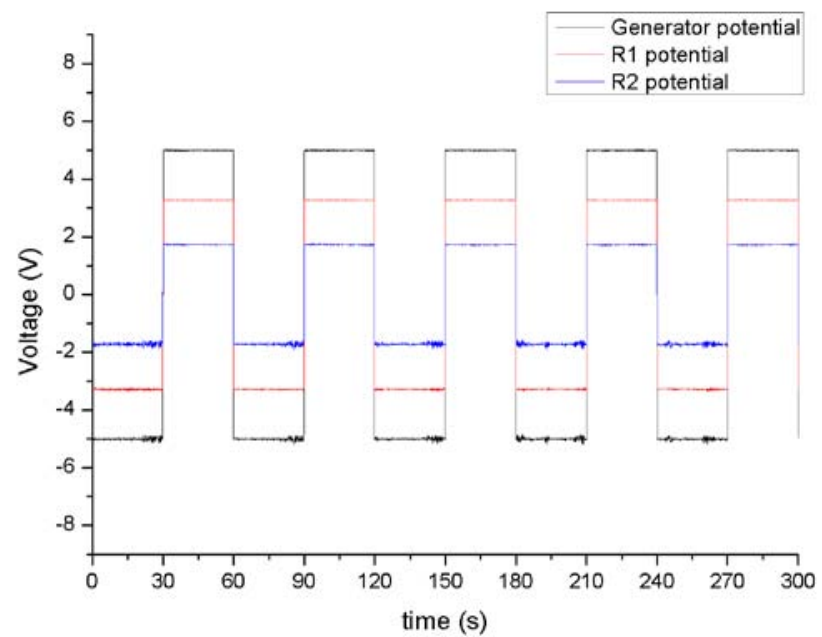

Figure 5: Potentials with a constant resistance

\section{Resistance variation of the electrolyte}

In order to evaluate the influence of time on the electric resistance variations of the electrolyte, $\pm 5 \mathrm{~V}$ is applied across the electrolyte structure for 30 seconds for a total measurement of 50 minutes. The constant resistance of the circuit here is $R=1.435 \mathrm{M} \Omega$. Considering that the values obtained for the positive applied voltage are more regular than those obtained for the negative voltage, the resistance variations are exposed only for $\mathrm{a}+5 \mathrm{~V}$ potential application. The resistance of the electrolyte is observed to increase at every potential application, from $0.007 \mathrm{M} \Omega$ to 1.210 $\mathrm{M} \Omega$ at the first cycle and from $0.009 \mathrm{M} \Omega$ to $1.517 \mathrm{M} \Omega$ at the $50^{\text {th }}$ cycle. The average resistance of the electrolyte also increases during the entire measurement time, as seen in figure 6.

The average resistance of the electrolyte, as observed in figure 6 , increases $18 \%$ in 50 minutes, i.e. 50 cycles. Increase of resistance corresponds to a decrease in the conducting properties of the electrolyte. This phenomenon is traduced, in a physical way, by a slowed down movement of the electrons.

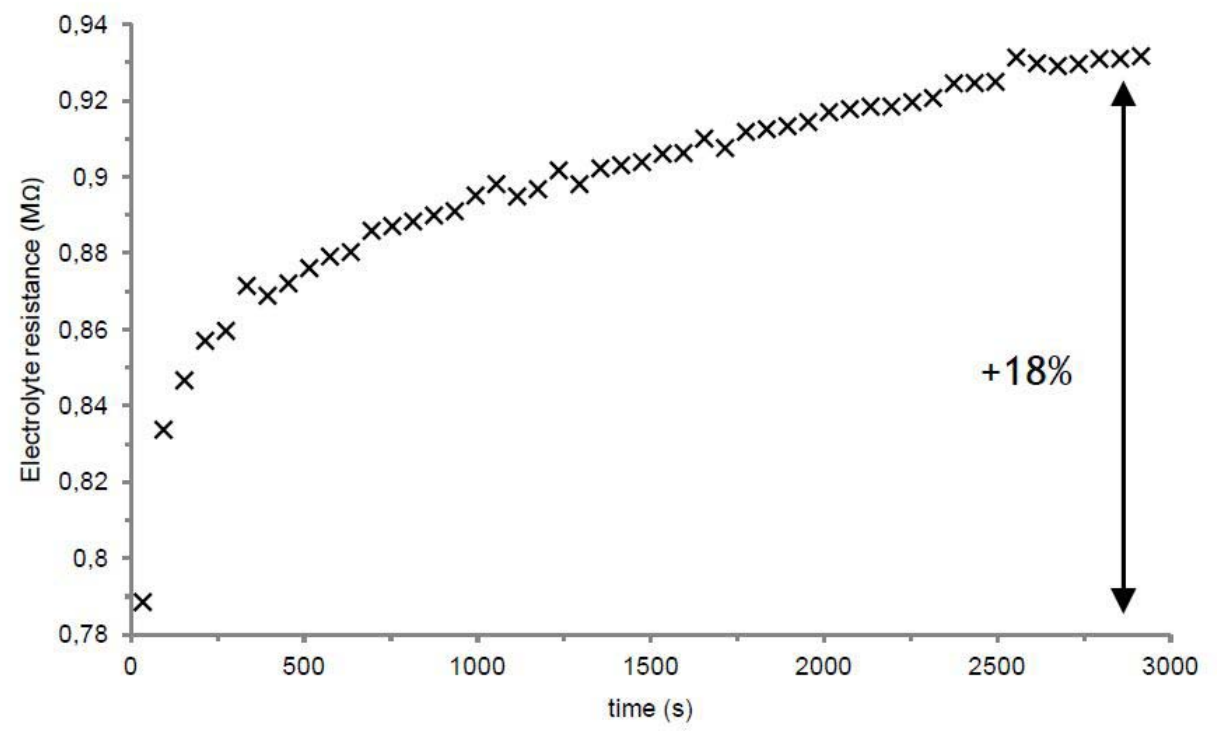

Figure 6. Electrolyte average resistance increase for 50 cycles 
Yet, the electrochromism colour change phenomenon is linked to the electron exchanges through oxidation and reduction. Consequently, the slowed down electron movement probably participates in the degradation of the colour change of the electrochromic textile structure.

Comparison between the electrolyte electric resistance increase and the electrochromic effect degradation

In order to evaluate the colour change of the electrochromic device, DeltaE CIE $L^{*}$, $a^{*}$ and $b$ is calculated between the initial oxidised colour and the successively obtained oxidised colours. Figure 7 presents the DeltaE CIE L*, $a^{*}$ and $b^{*}$ average percentage increase for 50 cycles. As observed, the initial green colour of the electrochromic device considerably changes with time and number of cycles. After 900 seconds, representing 15 cycles, the obtained colour stabilises. After 50 cycles, the oxidised state of the electrochromic device, which has been observed as darker, redder and less intense, has a difference of $900 \%$ in DeltaE CIE $L^{*}, a^{*}$ and $b^{*}$ with the initial oxidised colour.

While the electrolyte resistance increases, the colour difference also increases. Increase of resistance corresponds to a decrease in the conducting properties of the electrolyte. This phenomenon is traduced, in a physical way, by the slowed down movement of the electrons. Yet, the electrochromism colour change phenomenon is linked to the electron exchanges through oxidation and reduction. Consequently, the slowed down electron movement probably participates in the degradation of the colour change of the electrochromic textile structure.

\section{Conclusions}

A method to measure and calculate the variation of the resistance of an electrolyte applied on a textile substrate has been explicated. Similarities between the electrical performance of the electrolyte and capacitor have been identified. The total charging and discharging time of the electrolyte is approximately 3 minutes. Resistance of the electrolyte increases when a voltage is applied. After 50 cycles with an applied potential of \pm 5 $\mathrm{V}$, the mean resistance of the electrolyte increases from $20 \%$. Resistance increase corresponds to a conductivity decrease. At the same time, the colour of the electrochromic device is observed to be altered. The initial oxidised colour of the device considerably changes in 50 cycles. A link can probably be made between the electrolyte conductivity decrease and the degradation of the electrochromic effect of the device. Indeed, electrochromism is allowed by exchange of ions and electrons. Hence, slowed down electron movements can logically limit the colour change of the electrochromic device. Further studies may then explore the parameters causing this conductivity loss.

\section{Acknowledgements}

This work has been undertaken with the support of project ANR - Homo Textilus (ANR-11-SOIN-007). This study was first presented during the $4^{\text {th }}$ ITMC International Conference (October 2013).

\section{References}

[1] Scarlet R., Deliu R., and Manea L.R., Smart textile systems. Solutions for different applications. $7^{\text {th }}$ International Conference TEXSCI 2010, Liberec, Czech Republic, September 6-8.

[2] Bamfiel P. and Hutchings M.G., Chromic Phenomena, Technological Applications of Colour Chemistry 2010: Royal Society of Chemistry Publishing. 562.

[3] Monk P.M.S., Mortimer R.J., and Rosseinsky D.R., Electrochromism : Fundamentals and Applications. VCH ed1995. p. 243-243.

[4] Monk, Mortimer and Rosseinsky, Electrochromism and

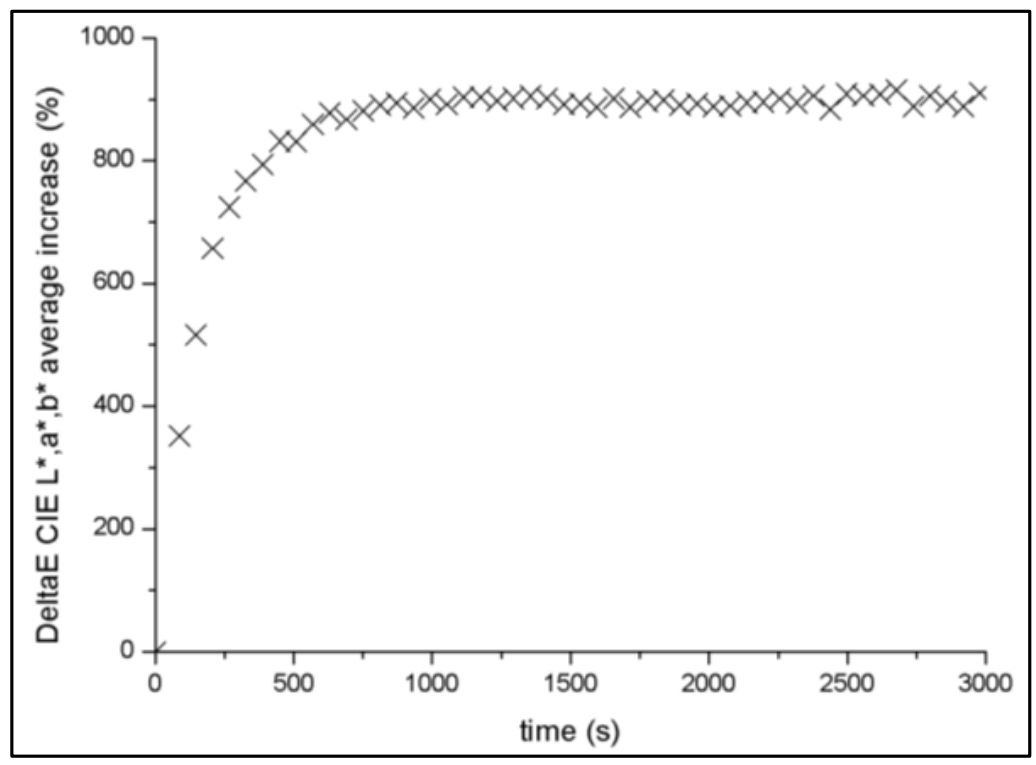

Figure 7. DeltaE $C I E L^{*}, a^{*}$ and $b^{*}$ average percentage increase for the oxidised states 
Electrochromic Devices. Cambridge 2007. 1-512.

[5] Granqvist C.G., Oxide electrochromics: Why, how, and whither. Solar Energy Materials and Solar Cells, 2008. 92(2) p. 203-208.

[6] Amb C.M., Dyer A.L., Reynolds J.R., Navigating the Color Palette of Solution-Processable Electrochromic Polymers. Chemistry of Materials, 2011. 23(3) p. 397-415.

[7] Granqvist C.G., Handbook of Inorganic Electrochromic Materials. Elsevier ed2002. p. 1-651.

[8] Cochrane C. et al., Flexible displays for smart clothing: Part I - Overview. Indian Journal of Fibre \& Textile Research, 2011. 36(December) p. 422-428.
[9] Kelly F.M. et al., Polyaniline: Application as solid state electrochromic in a flexible textile display. Displays, 2013. 34(1) p. 1-7.

[10] Meunier L. et al., Flexible displays for smart clothing : Part II - Electrochromic displays. 2011. 36(December) p. 429435.

[11] Ding Y. et al., A simple, low waste and versatile procedure to make polymer electrochromic devices. Journal of Materials Chemistry, 2011. 21(32) p. 11873.

[12] Panzer Matthew J., Frisbie Daniel C. Exploiting Ionic Coupling in Electronic Devices: Electrolyte-Gated Organic Field-Effect Transistors. Advanced Materials, 2008: p. 3177-3180. 